\title{
OBSERVATIONS \\ ON PULSATING AURORAS
}

is

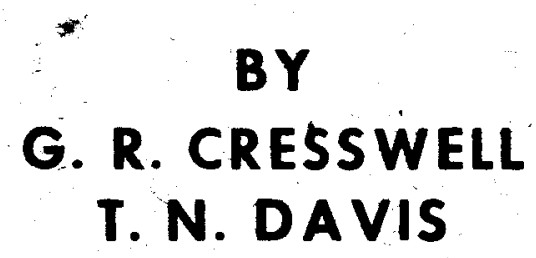

GPO PRICE

CFSTI PRICE(S) $\$$

N66 24686

Hard copy $(\mathrm{HC}) \frac{100}{150}$
Microfiche (MF)

ก 653 July 65
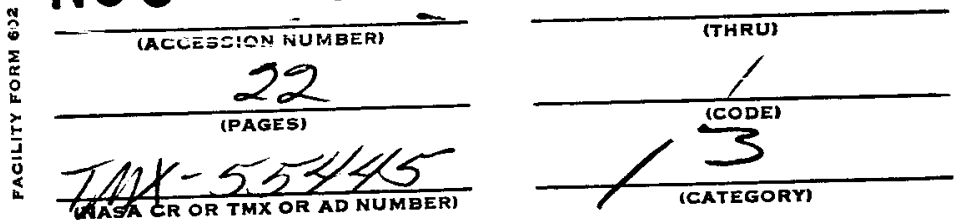

NASA

NOVEMBER 1965 


\title{
Observations on Pulsating Auroras
}

\author{
G. R. Cresswe11
}
Geophysical Institute of the University of Alaska
College, Alaska
and

T. N. Davis*

Goddard Space Flight Center

Greenbelt, Maryland

ABSTRACT

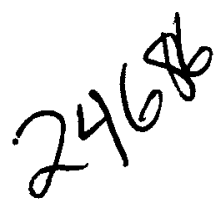

Observations on pulsating auroras occurring near the equatorward boundary of auroral displays during their post-breakup phase have been made with an image orthicon television system and with photometers. It is found that the pulsating phase of the display is composed of numerous relatively short sequences of pulsations which are restricted to small field-aligned volumes. The luminosity varies synchronously within a given auroral form but adjacent forms may pulsate asynch ronous $1 \mathrm{y}$.

\section{Introduction}

Pulsating auroras are characterized by their quasi-periodic intensity variations, their eastward drift and their low intensity. According to Georgio (1962) the 5577A intensity of pulsating auroras never exceeds $3 \mathrm{kR}$ (kilorayleigh). The regularity of the luminosity variations of the pulsating aurora distinguishes it from other auroral types which also may vary rapidly in luminosity but never in a periodic or quasi-periodic way. The visual observer most readily recognizes pulsating auroral forms having luminosity variations with a periodicity of 1 to 10 or more seconds. To him individual forms often appear to blink on and off as the level of luminosity passes through his visual threshold (about $1 \mathrm{kR}$ at wavelength 5577 A). The visual observer also recognizes the fact that pulsating patches in different parts of the sky do not pulsate synchronously (Parsons and *Now at Geophysical Institute of the University of Alaska, College, Alaska. 
Fenton, 1953 and Georgio, 1962). Photometrically, pulsations have been observed in the period range 0.1 second to 1 minute.

Within the complicated normal auroral display the pulsating aurora stands out as a special type apparently limited in latitude and local time. This restricted spatial and temporal variation plus the periodic nature of its luminosity variation makes the pulsating aurora an especially appealing phenomenon for detailed study. Here we present some observational results obtained from the application of narrow field photometers and an image orthicon television system.

Latitudinal Distribution. Using visual observations taken from College during 1950-1952 Heppner (1954) found that most pulsating auroras occurred between geomagnetic latitudes $62^{\circ}$ and $67^{\circ}$ with very few pulsating forms being observed north of that region. At least in part, the distribution found by Heppner might be attributed to the difficulty in detecting pulsating forms lying near the horizon. However, during ten days of visual observation and recording with an image orthicon television system at Fort Churchill (geomagnetic latitude $68^{\circ}$ ) during October 1963 we did not observe any pulsating aurora overhead even though some major auroral displays occurred. Another partial substantiation of Heppner's result comes from all-sky camera observations. Although luminosity variations in this frequency range are not detected by the all-sky camera, one can usually recognize the types of auroral forms that may show pulsations and these are not often observed on al1-sky films taken at the higher-latitude stations, with the exception that they may occur over a station located just poleward of the auroral zone when that station is on the day-side of earth (Davis and DeWitt, 1963). The pulsating forms tend to occur toward the equatorward boundary of the auroral display; this fact is in agreement with the latitudinal distribution described above and indicates that pulsating auroras will tend to occur at lower latitude as the level of global activity increases. 
Temporal Distribution. Pulsating auroras occur during and perhaps after the recovery phase of negative bays in $\mathrm{H}$ observed at stations lying near or equatorwards of the peak of the auroral zone. Thus the pulsating forms are rarely if ever observed in early evening and have their maximum occurrence in the few hours following local geomagnetic midnight (cf. Heppner, 1954). Pulsating forms frequently are observed until they are obscured by daylight and their occurrence rate on the day-side of the auroral zone is unknown.

General Appearance of the Sky during Pulsating Aurora. Much of the data presented here was obtained on the local morning of March 17, 1964 at College, Alaska within the period indicated by al1-sky photographs contained in Figure 1a. The pulsations on this morning began near 0035 and continued for several hours; the appearance of the sky during this period of pulsating aurora is typical. As seen on Figure la the aurora during this interval consisted mainly of diffuse arc-like forms and some patches; note that the overall alignment during part of this periud differs markedly from the east-west orientation commonly appearing during most of the normal auroral display. A copy of the College insensitive magnetogram $\mathrm{H}$ trace appears in Figure $1 \mathrm{~b}$.

\section{Observations}

Instrumentation. The image orthicon television system used for these observations has a $14^{\circ}$ field of view through an $\mathrm{f} / 0.75$ field lens; $1 / 60$ second exposures are recorded on film at the rate of 24 per second. An S-10 photocathode surface on the image orthicon target allows a peak sensitivity at $4500 \mathrm{~A}$ with $10 \%$ of peak sensitivity being near 3200 and $6900 \mathrm{~A}$. This instrument has more contrast and has somewhat more sensitivity than the eye so that it can detect weaker auroral forms and provide more structural detail than the visual observer.

One of the photometers used here has four fields of view, each of diameter $1.5^{\circ}$; another has a single $1.5^{\circ}$ field of view. Blue glass filters with $50 \%$ 
transmission points at 3900 and $4550 \mathrm{~A}$ were placed before these instruments so

that they were most sensitive to $\mathrm{N}_{2}^{+}$First Negative band emissions.

Types of Pulsating Aurora. With the television observations it is possible to distinguish several types of pulsating forms on the basis of their shapes. Since practically all of the television observations were taken in the magnetic zenithal region, the shape is that seen from below looking approximately up along the magnetic field lines. The types so far observed are:

1. Thin $(0.2-2 \mathrm{~km})$ sharply-defined arcs. These may be oriented in any direction but are often approximately east-west.

2. Irregularly shaped forms several kilometers in extent and having internal detail.

3. Shapeless regions several or more kilometers across showing no internal structure. Typically these are very weak and fluctuate rapidly with period down to $0.2 \mathrm{sec}$. They are sub-visual.

4. Flaming aurora. These are undetectable as such when observed from the base of the magnetic field line on which they appear. However, seen obliquely to the field line they appear to be field-aligned and decrease or increase in luminosity through the instrumental threshold first at some part on the form and then progressively upwards or downwards along the magnetic field to give the appearance of rising flames in a wood fire. An example is given in Figure 2 . There may be no difference between this category and category 1 above, or the flaming aurora may be a sub-class of category 1.

5. In addition to the above types there is a type of pulsating form easily detected visually: relatively large irregularly-shaped patches $50 \mathrm{~km}$ or more in horizontal extent which pulsate quasi-periodically at periods near $10 \mathrm{sec}$.

Luminosity Variations. Both the photometric data and the photometer tracings made from the television films show a variety in the types of temporal luminosity variation in the pulsating aurora, several examples of which are shown in Figure 3 . 
The complex temporal variations illustrated in Figure 3 are typical of all our data, and so far it has not been possible to determine definitely if the form types listed in the previous section have any characteristic temporal variation in luminosity.

Perhaps one of the most significant results of the study so far is that the nature of the observed luminosity variation of pulsating aurora critically depends upon the field of view of the observing instrument. An illustration of this finding is given in Figure 4. At $0105150^{\circ}$ West Meridian Time on March 17, 1964 two relatively thin pulsating arcs appeared within the field of view of the television system. The magnetic zenith separated the two arcs. Multiple photocells placed at various points on the projected image of one of the two arcs showed that each arc pulsated in phase with itself everywhere within the $14^{\circ}$ field of view. This is a general result: it is found without exception that all observable points on a pulsating form, when viewed from a magnetic field line occupied by the form, exhibit in-phase luminosity variation.

However, the two arcs shown in Figure 4 exhibit completely dissimilar temporal behavior. One starts with a burst-1ike pulsation which later becomes more sinusoidal but the period remains near $1.2 \mathrm{sec}$. The other auroral arc has more irregular and larger variations with periods ranging from 2 to $4 \mathrm{sec}$. The photocell tracing of the entire field of view (lower trace on Figure 4) is more akin to that of the brighter of the two forms (central trace on Figure 4) but far from identical. The weak sinusoidal variation of the first-mentioned arc is not detected in the full-frame photocell trace. Another example of asynchronous luminosity pulsations over a longer time interval is depicted in Figure 5. The dissimilarity of the photocell traces there indicates that the viewed portion of the pulsating display was made up of small forms pulsating asynchronously; visual observation of the television film verifies this conclusion.

As the photometric field of view is increased more asynchronously pulsating forms are observed and the resulting integration becomes more chaotic and difficult 
to interpret. Here another problem, that of viewing angle relative to the magnetic field, becomes important and can result in major differences between the appearances of pulsating forms viewed in the magnetic zenith and at lower elevation angles.

Simultaneous observations with narrow-field $\left(1.5^{\circ}\right)$ photometers directed at the zenith and $42^{\circ}$ north of the zenith also indicate that the apparent periodicities within the pulsating aurora depend upon the viewing aspect angle. Power spectra (Figure 6) determined from such data samples indicate that the relative power spectral density in the 0.1 to 1 second period range is much higher in the zenith region than in the region near zenith distance $42^{\circ}$. Both spectra have appreciable spectral density in the 1-20 second period range with the peaks lying near 8 seconds,but the zenith spectral density plot shows the greater complexity. The interpretation of these spectra is difficult, however, the spectra appear to be in agreement with the observation that the higher-frequency luminosity variations are observed most in the zenith regions where the narrowfield observations used to produce the power density spectra are most likely to result from variations in only one or two distinct auroral forms. Observations taken at low elevation angles are more likely to include several auroral forms in the field of view.

Sequences of Pulsations. Although the pulsating phase of the display often lasts for several hours, the data indicate that the pulsating phase is composed of numerous short sequences of pulsations lasting from a few seconds to several tens of minutes. The best criteria for identifying a particular pulsating sequence are the pulse shapes and periods, as, for example, on Panels 1,3 and 4 of Figure 3 and in Figure 4. Often, temporally overlapping sequences of pulsations are observed in addition to the spatial overlapping discussed in the previous section. Without question, part of the reason why short pulsation sequences are observed is that individual pulsating forms are drifting across the instrumental field of view. However, the television observations do show many examples 
of pulsating forms making an appearance well inside the field of view and then ceasing to exist before drifting from the field, hence it is reasonably sure that the entire sequence is being observed in such a case. This facet of the pulsating aurora seems to be quite important since it leads to the suggestion that whatever mechanism produces the pulsating form at a specific location is effective for a relatively short time.

Eastward Motions. A characteristic of the pulsating aurora is its primarilyeastward motion. Northward or southward components are usually minor and no westward drifting forms have been observed (see Akasofu, et al 1966). The eastward drift speed ranges up to $1000 \mathrm{~m} / \mathrm{s}$ and is commonly several hundred $\mathrm{m} / \mathrm{s}$. In making these speed measurements an altitude of $100 \mathrm{~km}$ is assumed.

One of the better examples of an irregular pulsating form drifting across the field of view of the television is reproduced in Figure 7 . The measured drift velocity of this form is plotted versus the time between pulsations on Figure 8. Also plotted on Figure 8 is a curve giving the relationship between the longitudinal (eastward) drift velocity and time of oscillation between mirror points for adiabatically moving electrons of given energies, where the mirror points are taken to be at altitude $100 \mathrm{~km}$ on the magnetic field line through College. Except for the datum point labeled "1" on Figure 8, the data points lie well to the right of the drift velocity versus time of oscillation curve for electrons moving adiabatically in the geomagnetic field. Thus it does not seem possible that the auroral pulsations could be caused by bunches of electrons partially precipitating at each mirror point unless a transverse (to $\bar{B}$ ) electric field influences their drift rate. A southward-directed electric field (in the northern hemisphere) leading to an approximately $300 \mathrm{~m} / \mathrm{s}$ increase in eastward drift is required for the case depicted in Figure 7, datum point 1 being ignored. In the polar ionosphere where B $\$ 0.6$ gauss the required southward electric field 
is given by $E=v_{d} B / 10^{3}=(0.3)(0.6) / 10^{3}=18 \mathrm{~V} / \mathrm{km}$. If mirroring particle

bunches were the source of the pulsating aurora, then probably each succeeding pulsation would appear somewhat broader in time and more weak as the particle bunch becomes diffused due to a finite spread of velocities and to mutual repulsion of the negative charges. However, no such effects appear in the data and it seems that another mechanism must be sought to produce the quasi-periodic auroral pulsations.

\section{SUMMARY AND CONCLUSIONS}

Pulsating aurora is a special type of aurora which is restricted primarily to the equatorward boundary of the auroral display and to the post-breakup phase when the local horizontal magnetic field is strongly negative or recovering from a negative bay.

In this study pulsating forms in the 0.1 to 100 second period range have been observed with an image orthicon television system and photometers. Both thin arc-like forms and irregularly shaped forms have been observed to pulsate. Whereas, the pulsation of an individual form is observed to be everywhere in phase (within the instrumental field of view), adjacent portions of the sky are observed to pulsate independently. Furthermore, the observed characteristics of the pulsating aurora appear to depend strongly upon the viewing aspect angle relative to the local magnetic field. Another interesting characteristic of the pulsating aurora is its eastward drift typically at speeds of $100-1,000 \mathrm{~m} / \mathrm{s}$. The overall pulsating phase of the auroral display may last for several hours and appears to be composed of relatively short sequences of a few to several tens of individual pulsations, each restricted to a small field-aligned volume and lasting a few seconds to several minutes.

Due to its location on and near the equatorward boundary of the auroral display, the pulsating aurora very likely is caused by a mechanism acting along closed field lines and perhaps involving particles trapped in the outer fringe of the radiation be1t Tho responsible mechanism evidently acts only when a negative 
bay is in progress, and most commonly when the negative bay is in its recovery phase. Since individual pulsating forms often are very restricted in space and time, some type of transient generation mechanism seems called for.

Acknowledgements

We are grateful to Dr. J. P. Heppner for his support and encouragement in this study and to Mr. A. E. Belon for many useful discussions. Part of the work at the Geophysical Institute was supported by NASA Contract NAS5-9065. 


\section{REFERENCES}

Akasofu, S.-I., C.-I. Meng, and D. S. Kimba11, Dynamics of the aurora VI. Formation of patches and their eastward motion, J. Atmospheric Terrest. Phys., in press, 1966.

Davis, T. N., and R. N. DeWitt, Twenty-four-hour observations of aurora at the southern auroral zone, J. Geophys. Res., 68, 6237-6241, 1963.

Georgio, N. V., Properties of auroral coruscations, Results of I.G.Y. Researches: Aurora and Airglow, No. 8, 17-20, 1962 (Also NASA Tech. Trans1. F-201).

Heppner, J. P., A study of the relationships between the aurora borealis and the geomagnetic disturbances caused by electric currents in the ionosphere, Thesis, California Inst. of Tech., 1954.

Parsons, N. R., and K. B. Fenton, Observations of the Aurora Australis, Macquarie Island, May 1950 - Apri1 1951, ANARE Interim Report No. 5, 1953.

Wentworth, R. C., W. M. MacDonald, and S. F. Singer, Lifetimes of trapped radiation belt particles determined by Coulomb scattering, Phys. Fluids, 2, 499-509, 1959. 


\section{Figures}

Figure 1. A. Reproductions of College all-sky pictures of $8 \mathrm{sec}$ exposure showing the general appearance of pulsating forms. The arrow marked $\mathrm{N}$ points to magnetic north; times shown are in $150^{\circ}$ West Meridian Time. B. Tracing of the $\mathrm{H}$ component of the College insensitive magnetogram.

Figure 2. The first of 4 observed cycles of a flaming aurora. The negative frames shown were taken at intervals of $1 / 16 \mathrm{sec}$; numbers below each frame here and in the following diagrams are frame numbers ( 24 frames per sec). This flaming form drifted eastward at $550 \pm 100 \mathrm{~m} / \mathrm{s}$; the apparent speed of the flames "moving" up the field was approximately $70 \mathrm{~km} / \mathrm{s}$, this value being very uncertain. The last frame shows the reappearance of a portion of the auroral form during the interval indicated.

Figure 3. Photometer tracings made viewing the full frame (approximately $14^{\circ}$ field of view) of the television films. Minimum and maximum intensities shown are less than 1 and several $k R$, respectively; increasing time left to right. Panel 1: Major fluctuations of period near six seconds on which one-second variations are superimposed. Panel 2: Roughly 0.5 second variations upon a slowly varying luminosity level. Panel 3: Large irregular bursts of luminosity occurring approximately five seconds apart. Panel 4: Smooth pulses of the same shape; each pulse lasts approximately two seconds and the repetition period is 7 to 10 seconds. In this example the pulses tend to occur less frequently as time progresses. Panel 5: Irregular 
pulses with variable shape and duration on which are superposed fluctuations of periods less than 0.5 seconds.

Figure 4. Photometric tracings made from projected television film with photocells placed on the images of an arc southwest (upper trace) and northeast (middle trace) of the magnetic zenith, respectively. The lower trace results from monitoring the full field of view with a photocell. The insert at lower right shows the field of view with its area at $100 \mathrm{~km}$ altitude and the placement of photocells on the images of the pulsating forms.

Figure 5. Photocell tracings of six different portions of the television field of view as indicated in the insert at lower right. Time increases to the right and the location of the field of view is the same as in Figure 4.

Figure 6. Power spectra of two 4-minute samples of auroral pulsations from the same time interval 0355-0359 $150^{\circ}$ WMT on 1 December 1964. A) Spectrum from a photometer directed $42^{\circ}$ north of the College zenith; B) Spectrum from a photometer directed at the College zenith.

Figure 7. The eastward progression of a pulsating patch that pulsated on and off 6 times within the field of view. The 6 frames are from the middle of each "on" period. Arrows on the frames indicate points used for drift velocity measurements. The dark lower edges on these negative prints are instrumental.

Figure 8. Drfft velocity in $\mathrm{m} / \mathrm{s}$ versus time between pulsations for the pulsating aurora of Figure 7 . The smooth curve shows the drift 
velocity (left-hand scale and electron energy, right-hand scale)

versus time of oscillation, $\mathrm{T}_{\mathrm{O}}$, for electrons having an equatorial

pitch angle of $3^{\circ} 10^{\prime}$ and mirroring at $100 \mathrm{~km}$ on the $\mathrm{r}_{\mathrm{e}}=5.5 \mathrm{field}$

line; results of Wentworth et al (1959) were used in the calculations. 


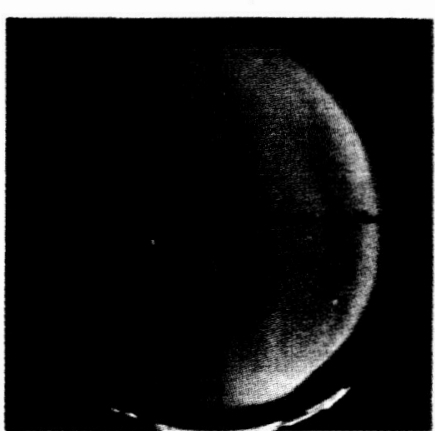

0015

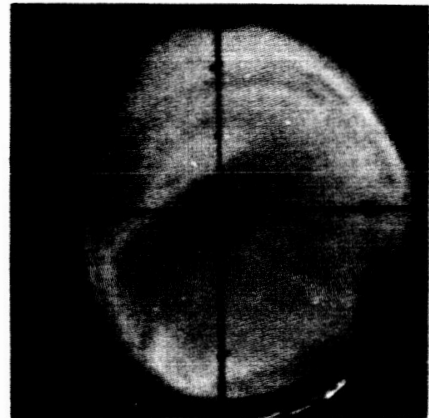

0100

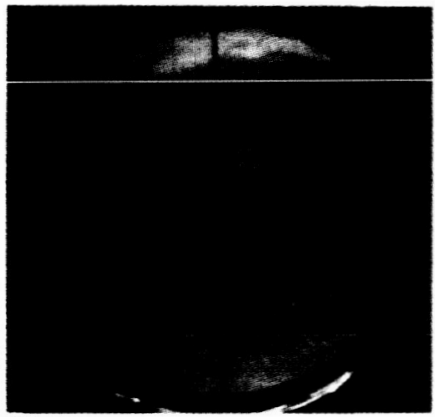

0145

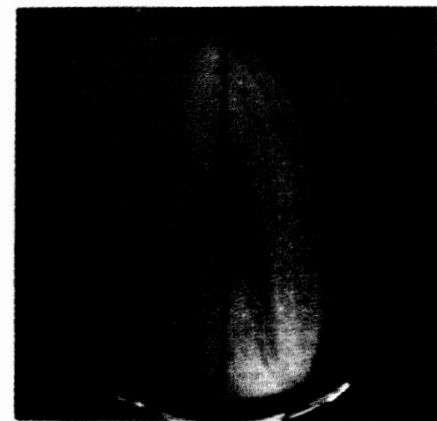

0030
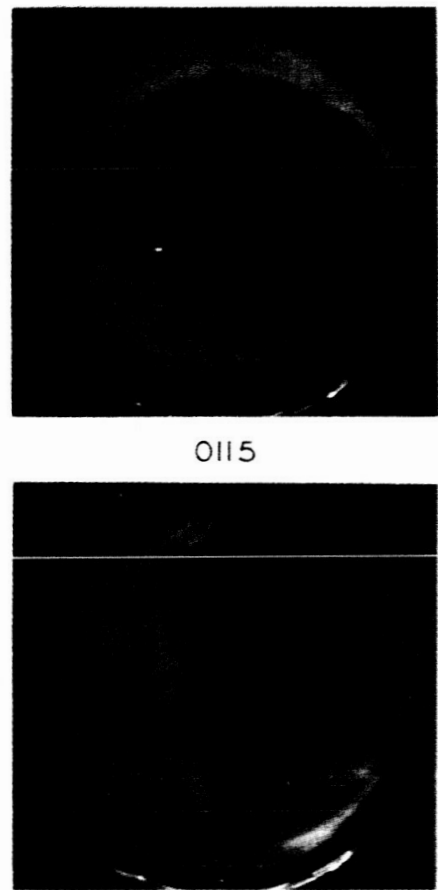

a)
0115

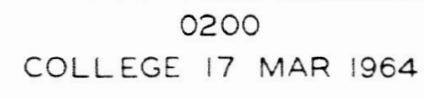

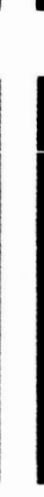

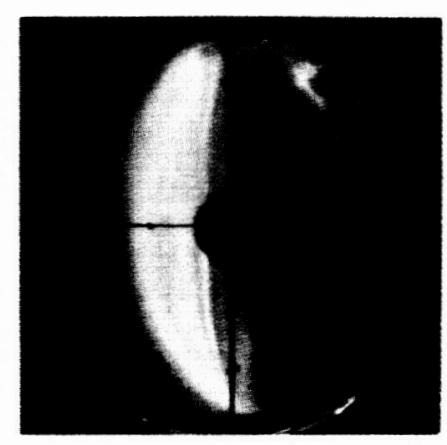

0045

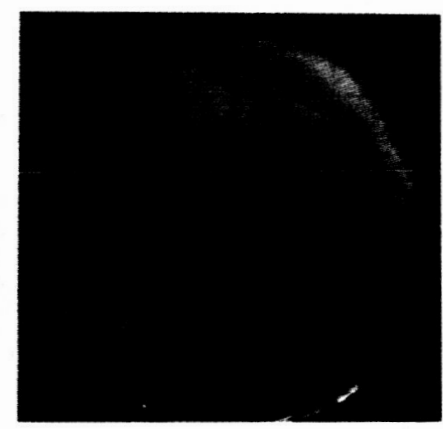

0130

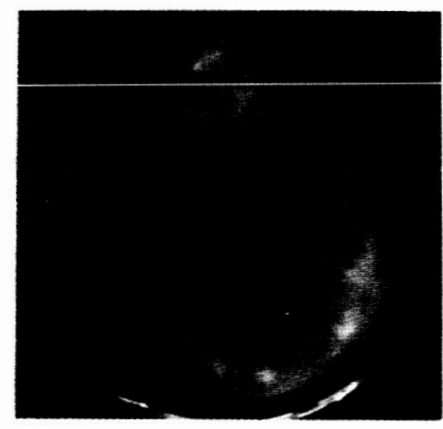

0215
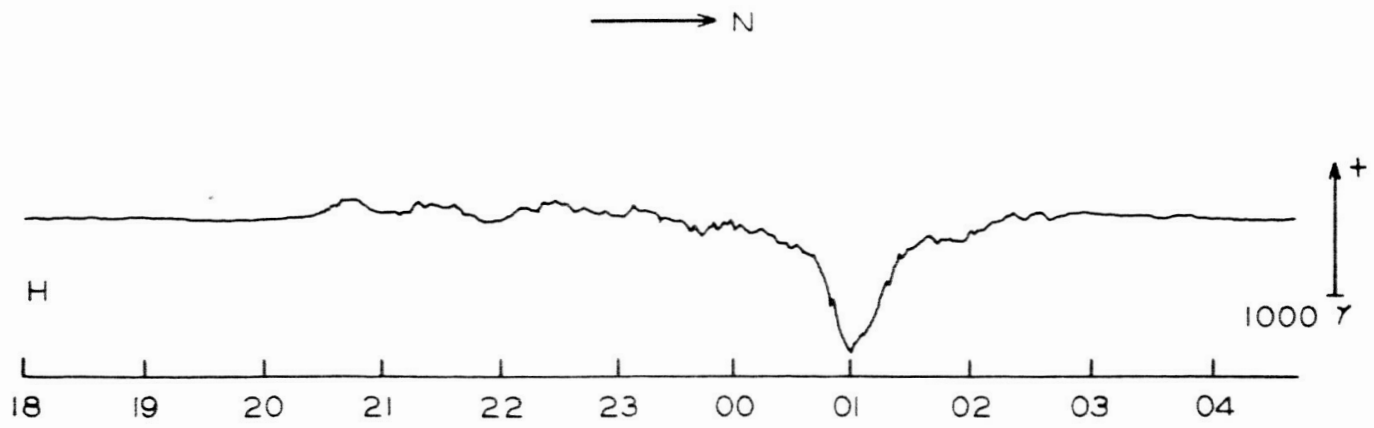

b) 


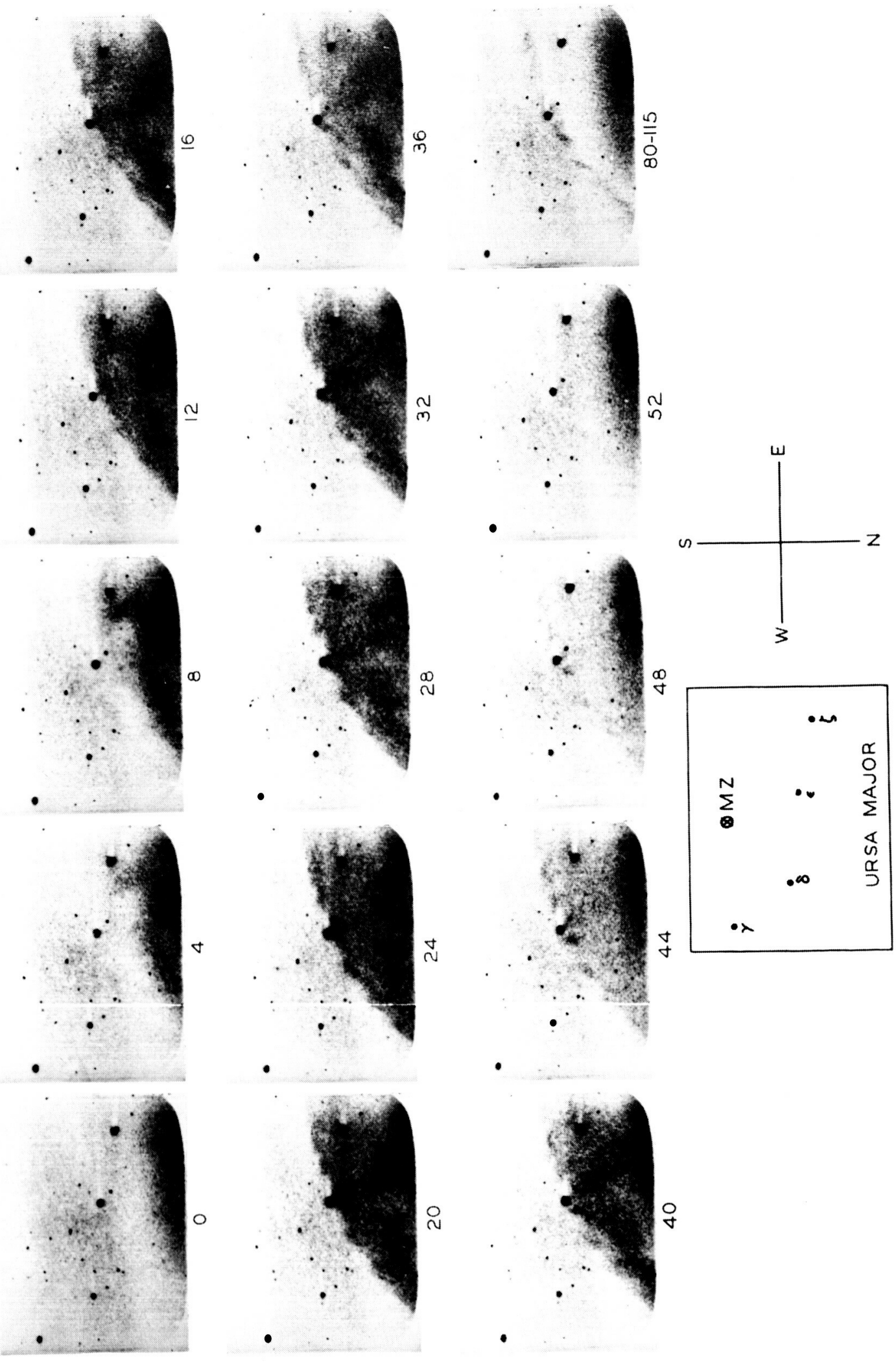

2
0
2
7
0
$0-1$
4 


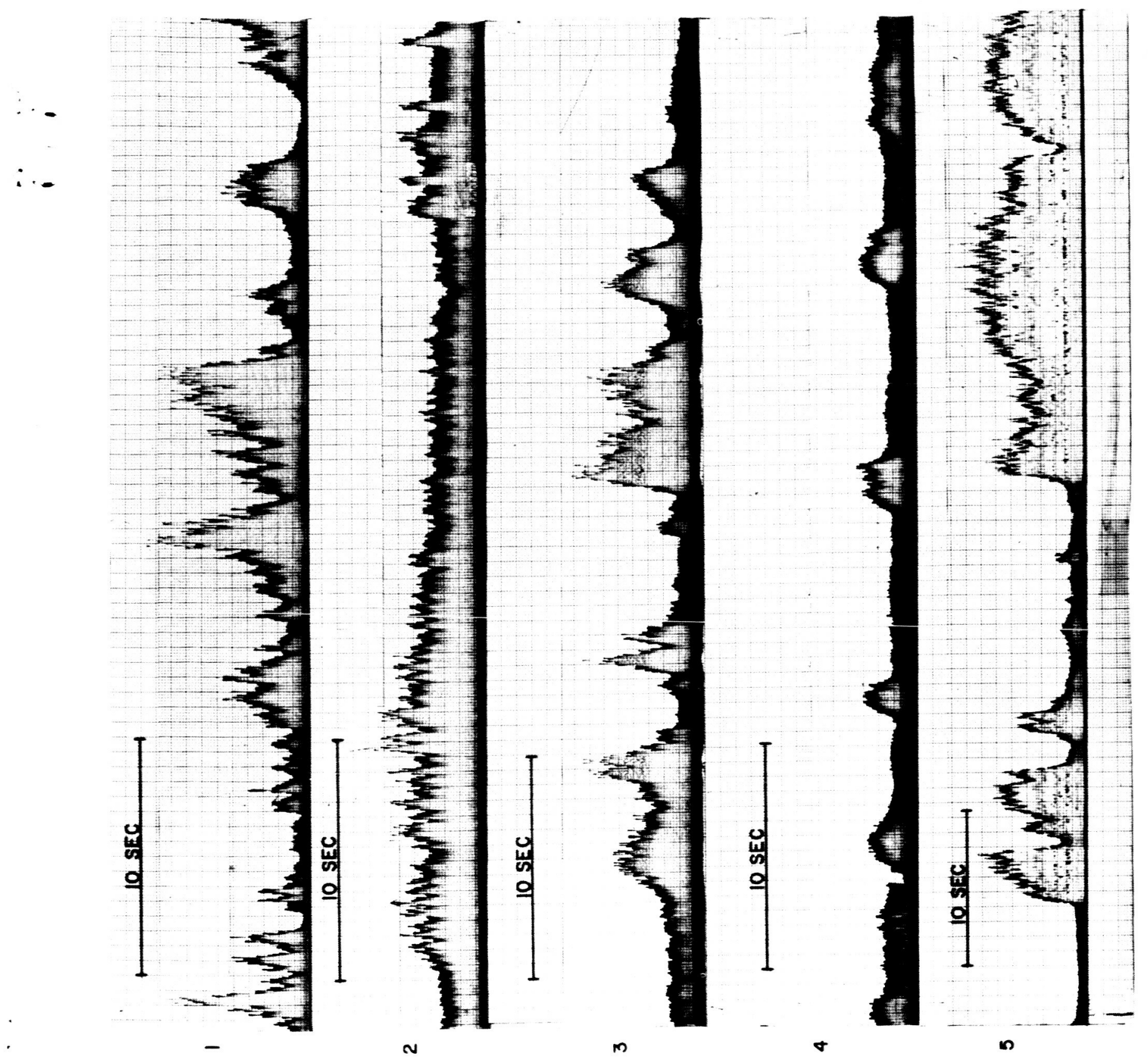

Figure 3 


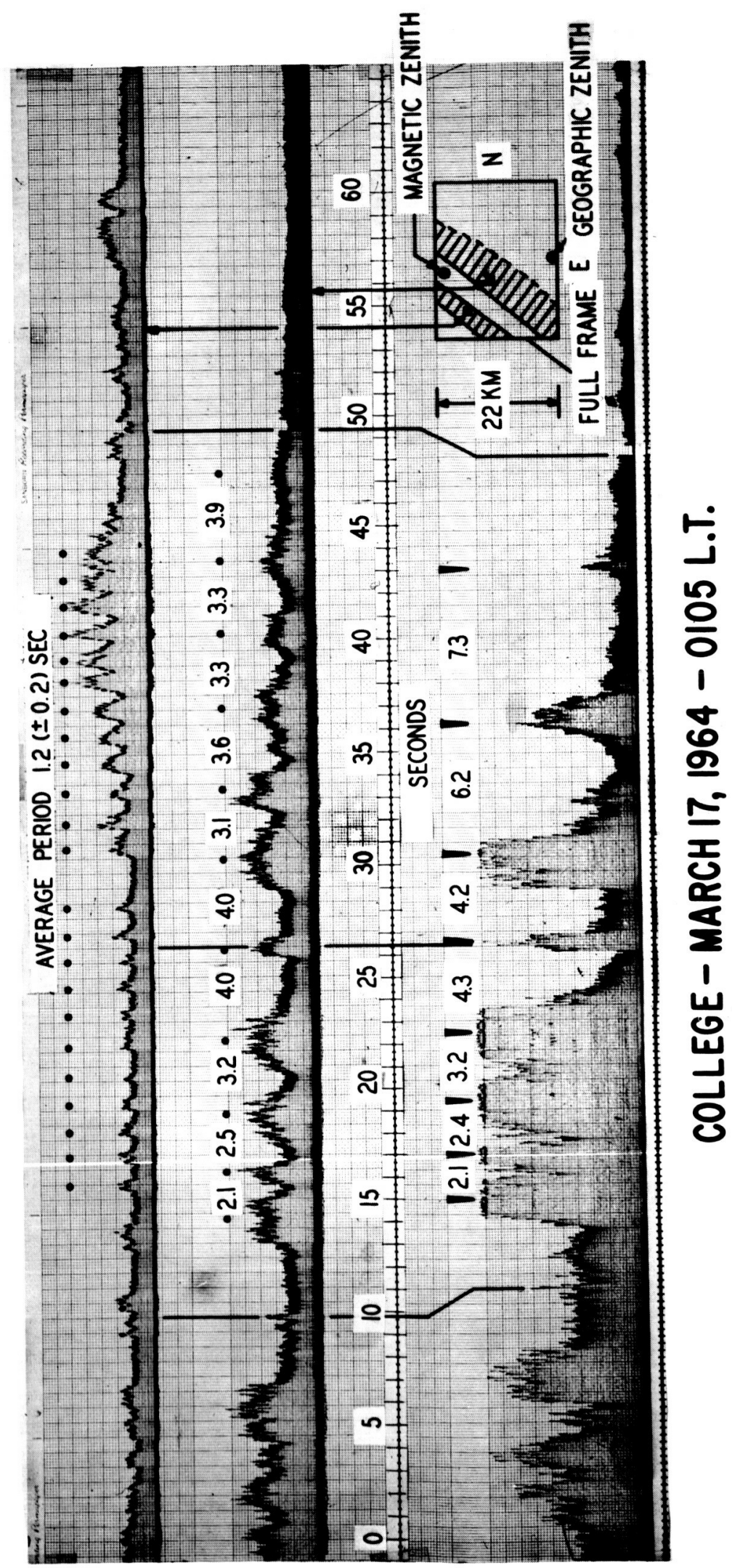

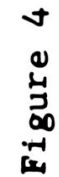




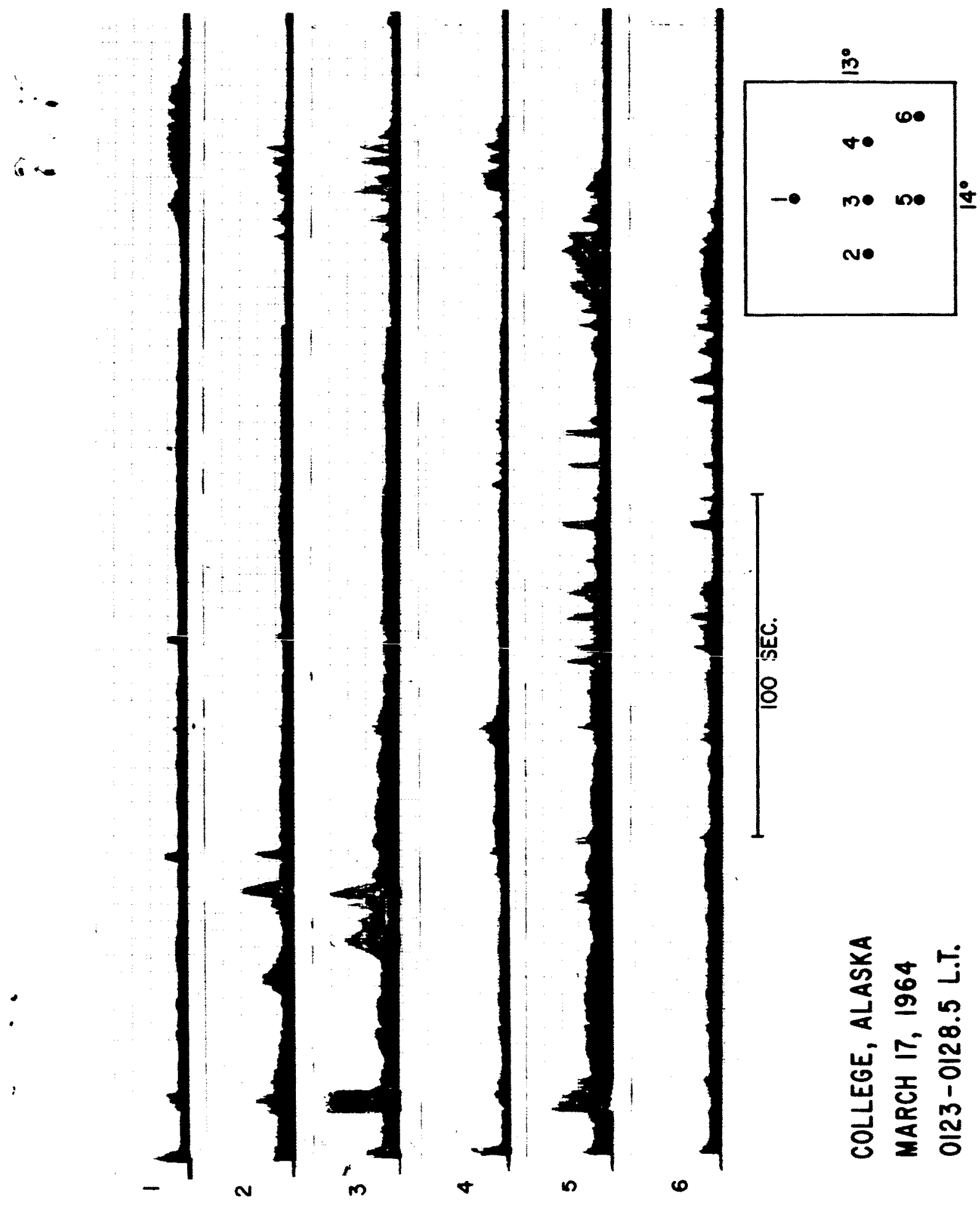

n 


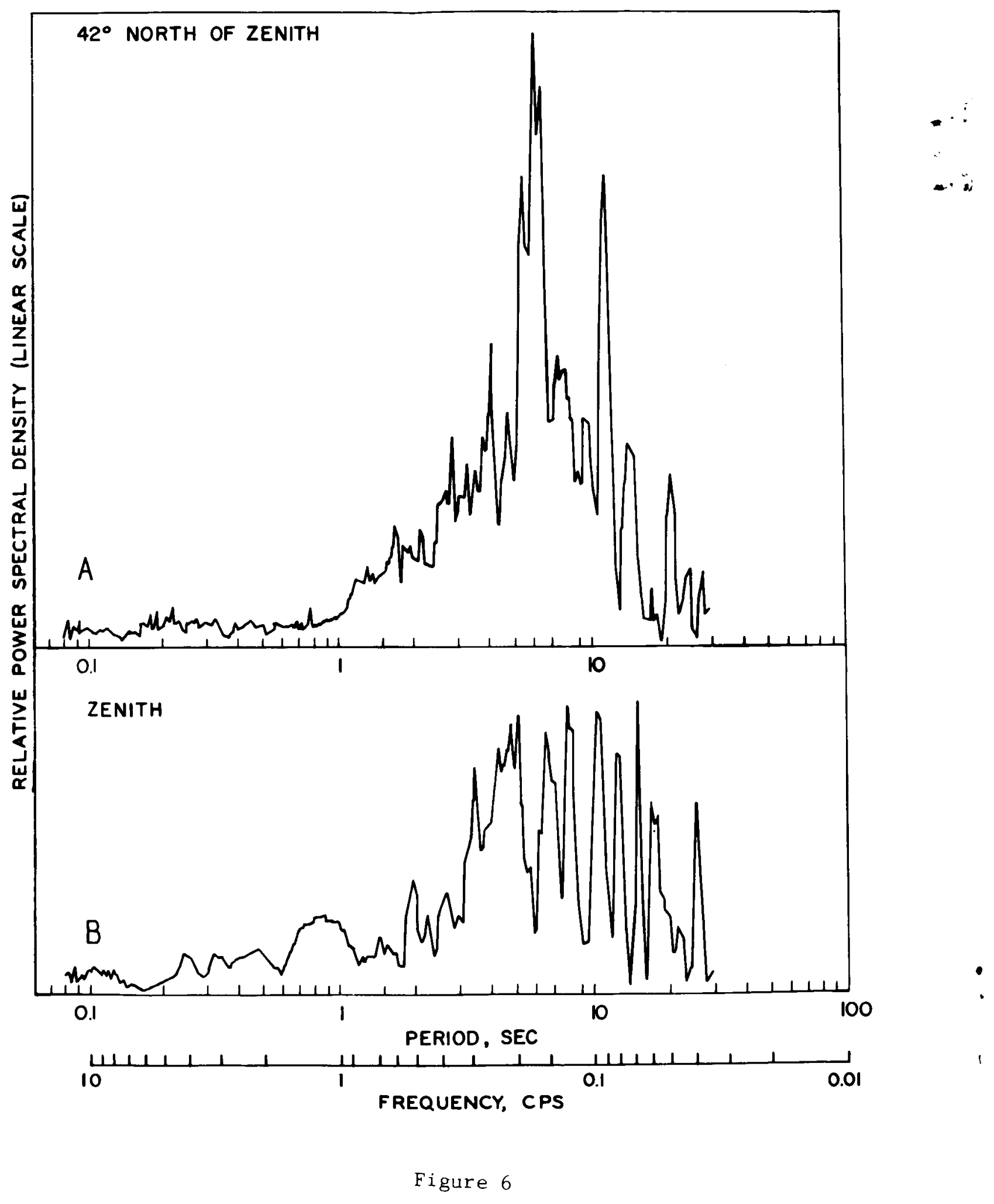



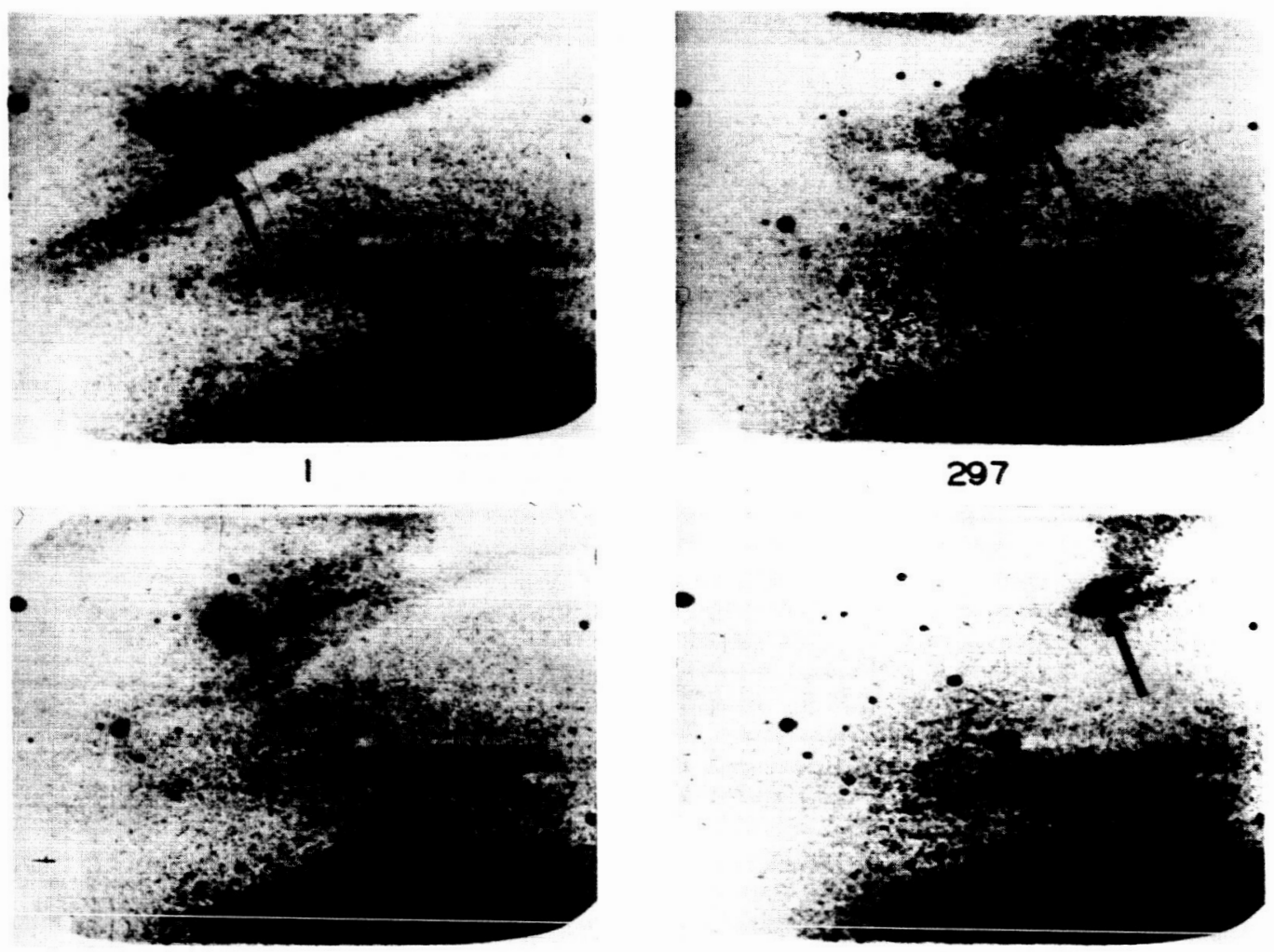

67

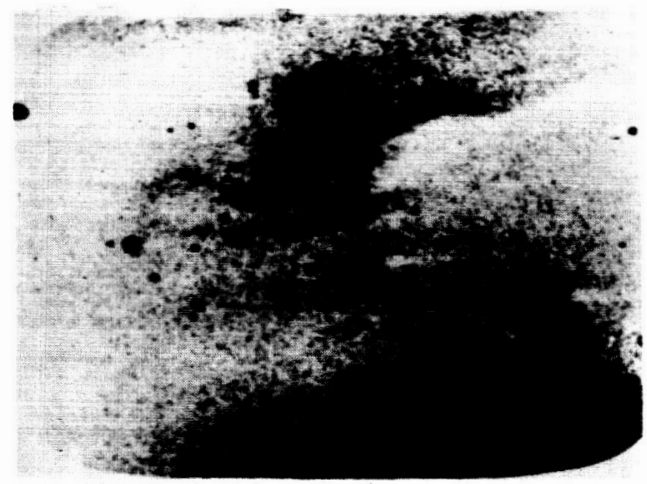

185
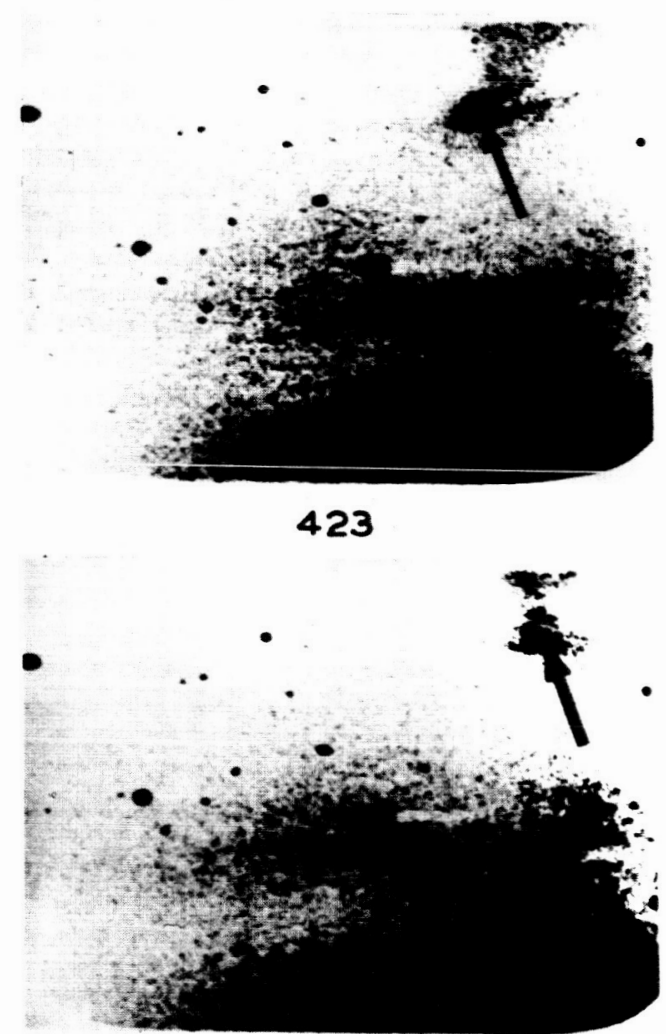

542

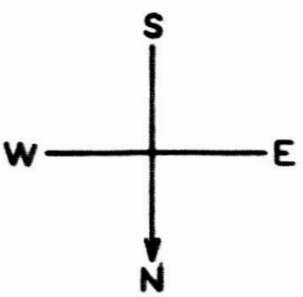

Figure 7 


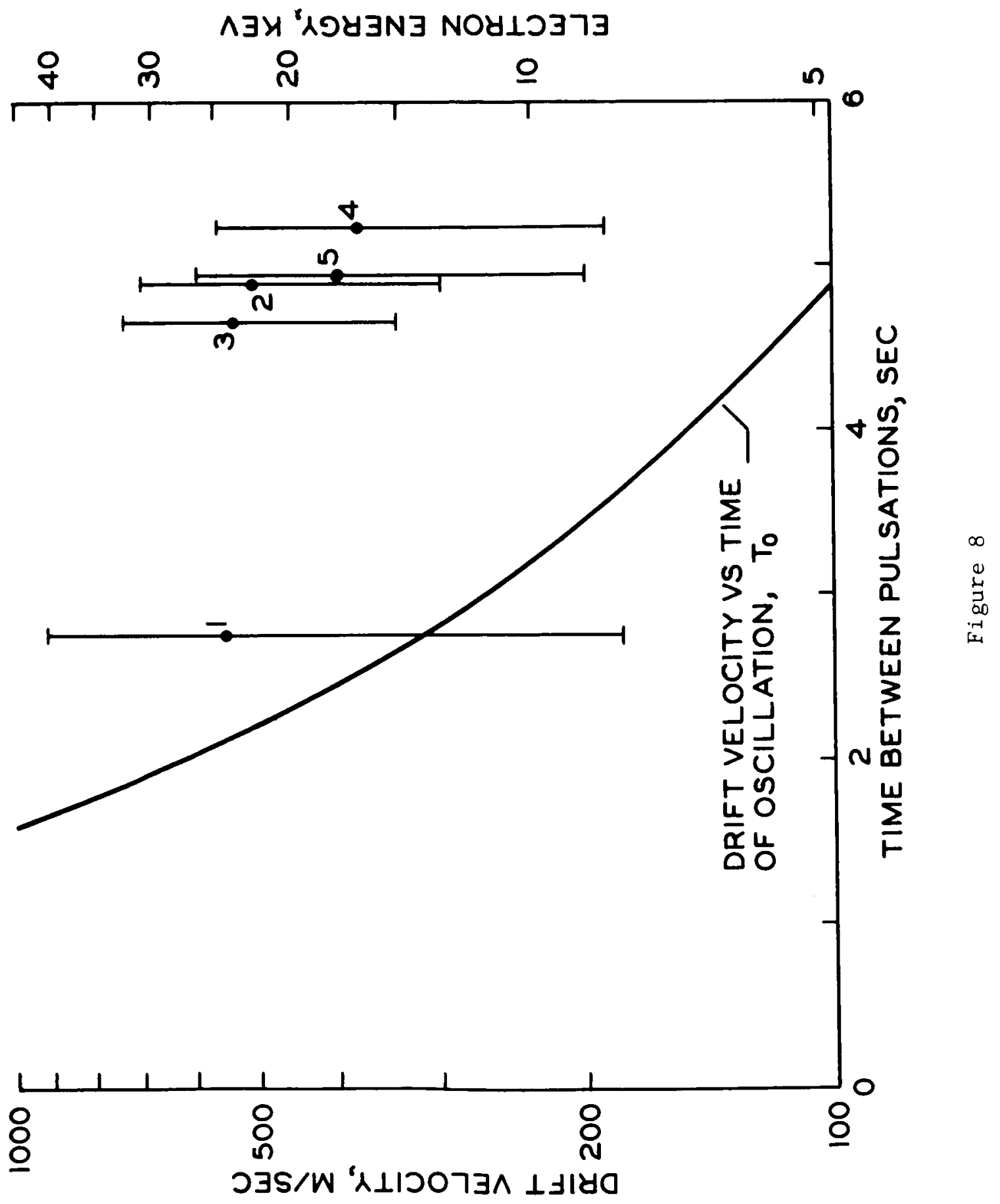

